Please do not remove this page

RMIT

UNIVERSITY

\title{
Power savings analysis of clipping and filtering method in OFDM systems
}

Gurung, Arun; Al-Qahtani, Fawaz; Sadik, Amin; Hussain, Zahir

https://researchrepository.rmit.edu.au/esploro/outputs/9921863901901341/filesAndLinks?institution=61RMIT_INST\&index=null

Gurung, A., Al-Qahtani, F., Sadik, A., \& Hussain, Z. (2008). Power savings analysis of clipping and filtering method in OFDM systems. 2008 Australasian Telecommunication Networks and Applications Conference, 204-208. https://doi.org/10.1109/ATNAC.2008.4783323

Published Version: https://doi.org/10.1109/ATNAC.2008.4783323

Repository homepage: https://researchrepository.rmit.edu.au

(c) 2008 IEEE. Personal use of this material is permitted. However, permission to reprint/republish this material for advertising or promotional purposes or for creating new collective works for resale or redistribution to servers or lists, or to reuse any copyrighted component of this work in other works must be obtained from the IEEE.

Downloaded On 2023/04/26 20:55:20 +1000 


\title{
Power Savings Analysis of Clipping and Filtering Method in OFDM Systems
}

\author{
Arun K. Gurung, Fawaz S. Al-Qahtani, Amin Z. Sadik, and Zahir M. Hussain \\ School of Electrical \& Computer Engineering, RMIT University, Melbourne, Australia \\ \{arun.gurung,fawaz.alqahtani\}@student.rmit.edu.au,amin.sadik@rmit.edu.au,zmhussain@ieee.org
}

\begin{abstract}
The clipping and filtering method is analyzed in terms of power savings. The analysis takes account of the gain in the amplifier efficiency due to Peak-to-Average-Power-Ratio (PAPR) reduction. Assuming a linear amplifier and a typical digital signal processor, the power savings is shown to be in the order of Watts.
\end{abstract}

-Keywords: Clipping and Filtering, Peak-to-Average-Power-Ratio (PAPR), Power Amplifier Efficiency, Orthogonal Frequency Division Multiplexing (OFDM).

\section{INTRODUCTION}

Orthogonal Frequency Division Multiplexing (OFDM), a popular multiplexing technique for many current and future broadband wireless communication systems, has principal drawback of high Peak-to-Average-Power-Ratio (PAPR). When passed through a non-linear power amplifier, 'peaky' OFDM signal generates in-band distortion which degrades the useful signal, and out-of-band radiation which leaks to and interferes adjacent channels. Amplifier back-off is necessary, but efficiency drops and more input power is required. Several PAPR reduction techniques have been proposed, an excellent overview of popular PAPR reduction techniques is given in [8][10].

Clipping amplitude [1] is stated as the simplest technique for PAPR reduction. As the occurrence of very high peaks is rare [9], the clipping method can produce peak reduction at small cost of system degradation. Conventional error correction codes can offset such small degradation [1][11][12]. Clipping is a non-linear operation, therefore distorts the OFDM signal. The out-of-band radiation is reduced by filtering. However, filtering makes peaks to regrow. Iterative clipping and filtering (ICF) [2][3] works in recursive fashion until target PAPR is obtained. Later its modified version such as Simplified Clipping and Filtering (SCF) [4] and One Iteration Clipping and Filtering (OICF) [13] schemes are shown to be less computation intensive. Clipping noise mitigation for performance improvement is also possible [14].

The effectiveness of clipping and filtering method has been assessed recently based on total degradation (TD) and results showed that it degrades rather than improves the system performance [5][15]. Yet, it is still a method of choice in recent $60 \mathrm{GHz}$ CMOS radio transceivers because of its simple implementation and reasonable PAPR reduction with small degradation [?].
In this paper, we assess the clipping and filtering method in terms of power savings. The main purpose of PAPR reduction is to decrease the back-offs and thus increase the amplifier efficiency. We show that how this efficiency gain translates into increased power savings. This investigation is motivated by the recent research on PAPR and amplifier model with efficiency [12][17][18][20][19].

Rest of the paper is organized as follows: Section II briefly explains the system model under consideration. General introduction on power savings due to PAPR reduction is given in Section III. Cost analysis as well as actual power savings for the clipping and filtering method are presented thereafter in Section IV. Section V summarizes the main outcome of the analysis and concludes the paper.

\section{SYSTEM DESCRIPTION}

\section{A. OFDM Signal}

Discrete-time OFDM signal can be written as,

$$
\begin{array}{r}
\mathrm{x}_{\mathrm{n}}=\mathrm{x}\left(\frac{\mathrm{nT}}{\mathrm{JN}}\right)=\frac{1}{\sqrt{\mathrm{N}}} \sum_{\mathrm{k}=-\mathrm{N} / 2}^{\mathrm{N} / 2-1} \mathrm{X}_{\langle\mathrm{k}+\mathrm{N}\rangle} \cdot \exp \left(\frac{\mathrm{j} 2 \pi \mathrm{nk}}{\mathrm{JN}}\right) \\
\mathrm{n}=0,1, \ldots \mathrm{JN}-1
\end{array}
$$

where $\langle\mathrm{k}+\mathrm{N}\rangle$ is $(\mathrm{k}+\mathrm{N})$ modulo $\mathrm{N} ; \mathbf{X}=\left\{\mathrm{X}_{0}, \mathrm{X}_{1}, \ldots, \mathrm{X}_{\mathrm{N}-1}\right\}$ represents input vector (of mapped symbols); $\mathrm{N}=$ number of subcarriers; $T=$ OFDM data symbol period; $\triangle \mathrm{f}=1 / \mathrm{T}$, frequency spacing for orthogonality; $\mathrm{J}$ is oversampling factor. $\mathrm{J}=1$ gives discrete-time signal sampled at Nyquist rate, whereas $\mathrm{J}=4$ provides sufficient samples to capture continuous-domain signal peaks [6]. The oversampled signal can be obtained by $(\mathrm{J}-1) \mathrm{N}$ zero-padding in the middle of the original input vector and taking IFFT of it. The zero-padded input vector looks like

$$
\mathbf{X}=\left\{\mathrm{X}_{0}, \ldots, \mathrm{X}_{\mathrm{N} / 2-1}, 0,0,0,0,0, \ldots, \mathrm{X}_{\mathrm{N} / 2}, \ldots, \mathrm{X}_{\mathrm{N}-1}\right\}
$$

\section{B. Peak-to-Average-Power Ratio (PAPR)}

The PAPR of discrete-time OFDM signal is written as

$$
\mathrm{PAPR}_{\mathrm{x}} \triangleq \frac{\max _{0 \leq \mathrm{n} \leq \mathrm{JN}-1}\left|\mathrm{x}_{\mathrm{n}}\right|^{2}}{\mathrm{E}\left\{\left|\mathrm{x}_{\mathrm{n}}\right|^{2}\right\}}
$$

where $\mathrm{E}\{$.$\} denotes expectation operator. PAPR is best de-$ scribed by its statistical parameter, complementary cumulative distribution function (CCDF). CCDF measures the probability of signal PAPR exceeding certain threshold $\gamma$ i.e. 


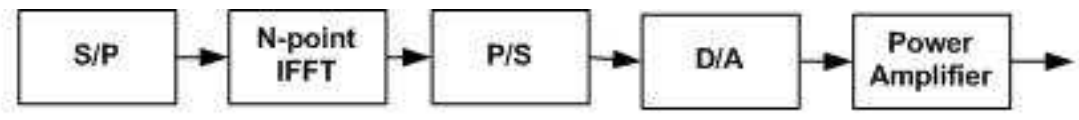

(a)

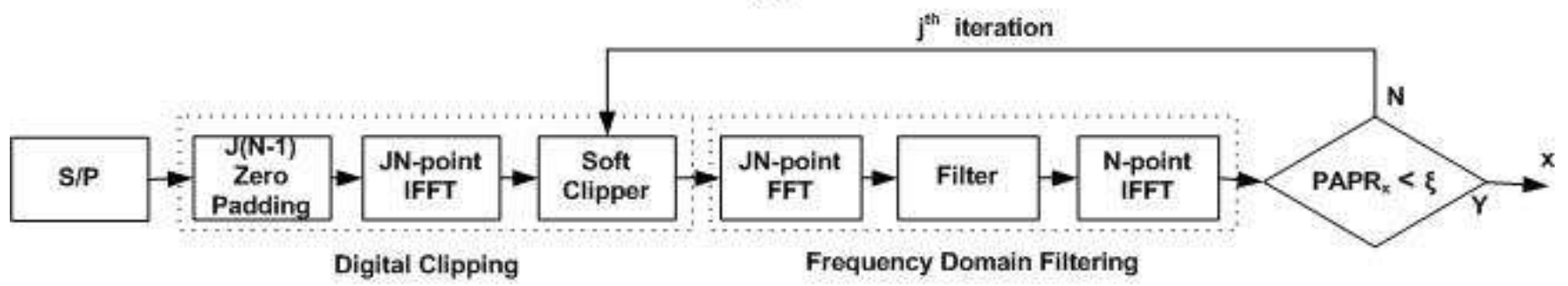

(b)

Fig. 1. OFDM Transmitter Block Diagram: (a) Original, and (b) With Clipping and Filtering

$\operatorname{Pr}\left[\mathrm{PAPR}_{\mathrm{x}}>\gamma\right]$. An approximated PAPR CCDF expression based on level crossing rate can be given as [9],

$$
\mathrm{p}(\gamma)=\mathrm{P}\left(\mathrm{PAPR}_{\mathrm{x}}>\gamma\right) \cong 1-\exp \left(-\mathrm{Ne}^{-\gamma} \sqrt{\frac{\pi}{3} \gamma}\right)
$$

and is accurate for a relatively high $\gamma$ and large number of subcarriers $\mathrm{N} \geq 64$.

$\gamma$ as a function of probability level $p$ can be written as [17],

$$
\gamma(\mathrm{p})=\frac{-1}{2} \mathrm{~W}\left(-6 \frac{\ln (1-\mathrm{p})^{2}}{\pi \mathrm{N}^{2}}\right)
$$

where W is Lambert's W-function defined by the inverse of $\mathrm{f}(\mathrm{W})=\mathrm{We}^{\mathrm{W}}$ [21]. Table I gives some $\gamma$ values as a function of $\mathrm{N}$ and p. They can also be obtained from the computer simulation curve, refer Fig (3).

TABLE I

$\gamma($ IN DB) AS A FUNCTION OF THE CLIPPING PROBABILITY AND THE NUMBER OF SUBCARRIERS

\begin{tabular}{cccccc}
\hline \hline$p$ & $N=64$ & $N=128$ & $N=256$ & $N=512$ & $N=1024$ \\
\hline $10^{-2}$ & 9.97 & 10.3 & 10.6 & 10.8 & 11.1 \\
$10^{-3}$ & 10.9 & 11.2 & 11.4 & 11.6 & 11.8 \\
$10^{-4}$ & 11.7 & 11.9 & 12.1 & 12.3 & 12.5 \\
$10^{-5}$ & 12.3 & 12.5 & 12.7 & 12.8 & 13.0 \\
$10^{-6}$ & 12.9 & 13.1 & 13.2 & 13.4 & 13.5 \\
\hline
\end{tabular}

As can be seen from the table, the threshold PAPR depends on clipping probability and number of subcarriers. In order to accurately assess the gain in power efficiency, we need to set a common benchmark which in our case is bit-error-rate (BER), BER depends on the clipping probability of a linear amplifier. Besides clipping probability, system BER is also a function of constellation size [22]. Higher the constellation size, more the errors with the same clipping probability and the number of subcarriers. To simplify power analysis, we take a reference clipping probability of $10^{-5}$.

\section{Power Amplifier and Efficiency}

Amplifier model is very important for power analysis. We assume a linear power amplifier (PA) model (equivalent to

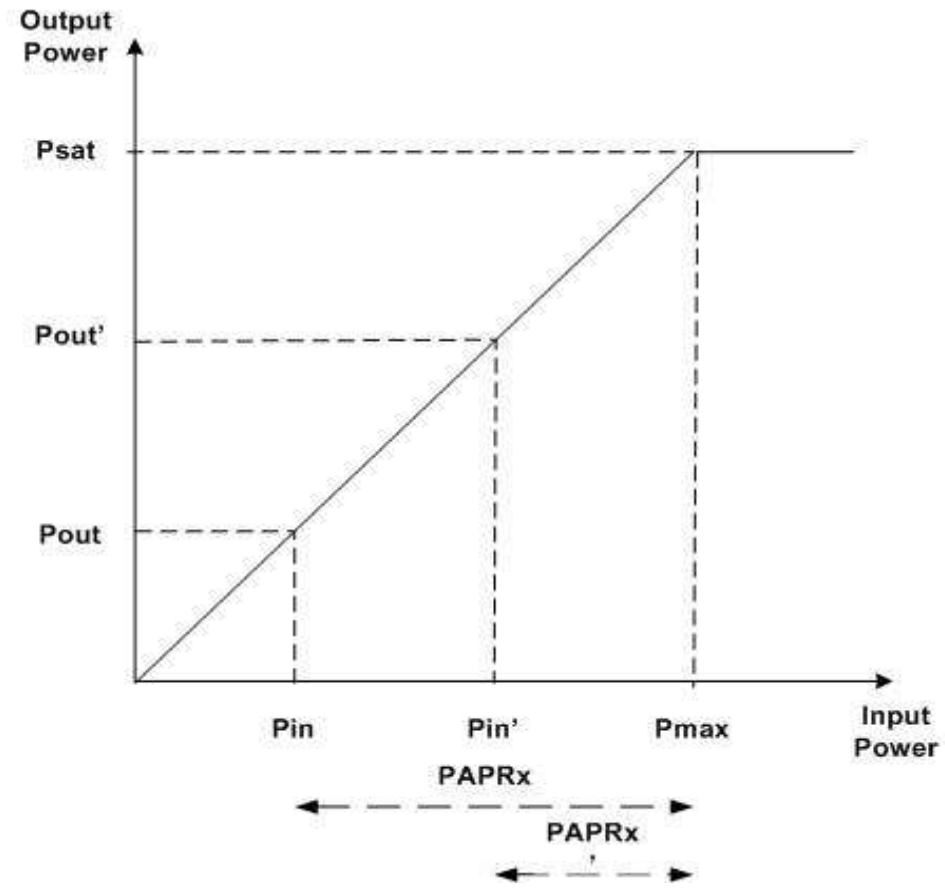

Fig. 2. A linear amplifier

ideally pre-distorted non-linear amplifier) as shown in the Fig (2). Input signal is amplified linearly until a certain level and thereafter is clipped to the output saturation level $\mathrm{P}_{\text {sat }}$. To optimize the amplifier operation, the input power is biased such that the maximum output power always remains around $\mathrm{P}_{\text {sat }}$ [24]. Appropriate amplifier back-offs need to be maintained to avoid excessive in-band distortion and spectral leakage. Back-offs are specified in terms of output back-off (OBO) or input back-off (IBO). OBO is the ratio of $\mathrm{P}_{\text {sat }}$ and average output power $\mathrm{P}_{\text {out }}$, whereas IBO refers to the ratio of input power corresponding to output saturation level, $\mathrm{P}_{\max }$ and average input power $\mathrm{P}_{\text {in. }}$. In a linear amplifier, $\mathrm{IBO}=\mathrm{OBO}$. With the maximum input power satisfying

$$
\max _{0 \leq \mathrm{n} \leq \mathrm{N}-1}\left|\mathrm{x}_{\mathrm{n}}\right|^{2}=\mathrm{P}_{\max }
$$


we can write $\mathrm{IBO}=\mathrm{OBO}=\mathrm{PAPR}$.

The efficiency of a PA is defined as,

$$
\eta=\frac{\mathrm{P}_{\mathrm{out}}}{\mathrm{P}_{\mathrm{dc}}}
$$

$\mathrm{P}_{\mathrm{dc}}$ being a constant amount of power consumed by the amplifier regardless of the input power. Class A PAs are the most linear amplifiers with maximum efficiency of $50 \%$ and its efficiency is given by $\eta=0.5 / \mathrm{OBO}$ [23]. Therefore, we can write the efficiency in terms of input signal PAPR as,

$$
\eta=\frac{0.5}{\mathrm{PAPR}_{\mathrm{x}}}
$$

To get an idea how inefficient would be the PA in the case of original OFDM signals, we take an example of $\mathrm{N}=256$ and $\mathrm{p}=10^{-5}$. From the Table I or Fig. 3, the input signal PAPR is $12.7 \mathrm{~dB}(=18.62)$ at which the amplifier has efficiency of only $0.5 / 18.62=2.68 \%$. Such a low efficiency, for instance, would drain the battery power very quickly. To prolong the battery life and save the system power, we need to seek to lower PAPR. Every 3dB PAPR reduction doubles the amplifier efficiency.

Combining equations (7) and (8), we get

$$
\mathrm{P}_{\mathrm{dc}}=2 \mathrm{P}_{\mathrm{out}} \cdot \mathrm{PAPR}_{\mathrm{x}}
$$

or equivalently

$$
\mathrm{P}_{\text {out }}=\frac{0.5 \mathrm{P}_{\mathrm{dc}}}{\mathrm{PAPR}_{\mathrm{x}}}
$$

The impact of the PAPR reduction on the power analysis can be seen in two ways.

1) When the average output power $P_{\text {out }}$ is fixed, and

2) When the supply power $P_{d c}$ is fixed.

Taking expectation of both sides in Equations (9) \& (10), average power trade-offs can be written as,

$$
\mathrm{E}\left[\mathrm{P}_{\mathrm{dc}}\right]=2 \mathrm{P}_{\text {out }} \cdot \mathrm{E}\left[\mathrm{PAPR}_{\mathrm{x}}\right]
$$

and,

$$
\mathrm{E}\left[\mathrm{P}_{\text {out }}\right]=\frac{0.5 \mathrm{P}_{\mathrm{dc}}}{\mathrm{E}\left[\mathrm{PAPR}_{\mathrm{x}}\right]}
$$

The PAPR reduction, thus, translates into either power savings when $\mathrm{P}_{\text {out }}$ is fixed or increased transmitted output power when $\mathrm{P}_{\mathrm{dc}}$ is fixed.

\section{Clipping and Filtering}

The signal peaks are clipped to a predetermined level. The clipped OFDM signal is represented as,

$$
\mathrm{x}_{\mathrm{c}}(\mathrm{n})= \begin{cases}\mathrm{x}(\mathrm{n}), & |\mathrm{x}(\mathrm{n})| \leq \mathrm{A}_{\max } \\ \mathrm{A}_{\max } \mathrm{e}^{\mathrm{j} \psi(\mathrm{n})}, & |\mathrm{x}(\mathrm{n})|>\mathrm{A}_{\max }\end{cases}
$$

$\psi(\mathrm{n})=\arg [\mathrm{x}(\mathrm{n})]$ represents phase of $\mathrm{x}(\mathrm{n})$. The phase of signal is preserved whenever signal exceeds clipping threshold $A_{\max }$. This type of clipping is referred to as Soft Limiting (SL). The clipping severity is quantified by clipping ratio $\zeta$ which is defined as the ratio of the threshold to the average signal power $\mathrm{P}_{\mathrm{i}}$ (that of before clipping),

$$
\zeta=\frac{\mathrm{A}_{\max }^{2}}{\mathrm{P}_{\mathrm{i}}}
$$

Note that clipping always reduces the average power of the signal. Therefore, the signal PAPR after clipping is always greater than $\zeta$. A relationship between the signal power before and after clipping (clipped signal + distortion) has been given in [12],

$$
\mathrm{P}_{\mathrm{o}}=\left(1-\mathrm{e}^{\zeta}\right) \cdot \mathrm{P}_{\mathrm{i}}
$$

which is accurate for large $\mathrm{N}$, i.e. when signal amplitude follows rayleigh distribution.

Ermolova [15] has derived an approximation for the ratio between power fallen in out-of-band region $\mathrm{P}_{\mathrm{o}, \mathrm{o}}$ and the average power of clipped signal (before filtering) $\mathrm{P}_{\mathrm{O}}$ as,

$$
\mathrm{p}(\zeta)=\frac{\mathrm{P}_{\mathrm{o}, \mathrm{o}}}{\mathrm{P}_{\mathrm{o}}} \approx 0.085 \cdot \exp \left(\frac{-\zeta}{\sqrt{2}}\right)
$$

$6 \mathrm{~dB}$ clipping results the ratio of $6.3 \times 10^{-3}(-22 \mathrm{~dB})$. Filtering is still necessary to attenuate out-of-band leakage further so that adjacent channels are not affected. Digital filtering such as scheme mentioned in [2] can be used. The clipped signal is converted into frequency domain by forward Fourier transform; only first N/2 and last N/2 components are taken and all other samples are set to zero; inverse Fourier transform of vector gives the filtered time-domain signal. Filtering makes peaks to regrow beyond the original clipping threshold, thus increasing the signal PAPR. In the iterative clipping and filtering (ICF) scheme the regrown pulses are clipped and filtered in an iterative fashion until target PAPR is obtained [2].

The system model under consideration is shown in Fig (1). The digital clipping and filtering blocks reside before digital-to-analog converter (DAC) and power amplifier. Pulse shaping is not considered so does cyclic prefix. Note that no change in receiver block is necessary for this scheme (unless performance improvement technique like described in [14] is used, here our focus is only on conventional scheme), therefore receiver block is not shown.

\section{Power SAVIngs ANAlysis}

The power savings directly relates to the signal PAPR: lower the PAPR, higher the savings. Computation of amplitude distribution after ICF is a bit tricky and to authors' knowledge no such analytical expression has been published to date. Researchers rather opt to use computer simulations. As such we have used empirical results for our analysis.

The Fig (3) shows the CCDF curves of 256 subcarriers modulated with QPSK symbols and oversampled by a factor of 5 . The time-domain signal is clipped at $\zeta=6 \mathrm{~dB}$, and subsequently filtered. Note that clipping of $6 \mathrm{~dB}$ or more induces negligible BER degradation [1][4]. Another two iterations of clipping and filtering were employed to clip down any peak-regrowths after filtering. 
From equations (9) or (11), we can write power savings as,

$\mathrm{P}_{\text {savings }}=\mathrm{P}_{\mathrm{dc}, \text { bef }}-\mathrm{P}_{\mathrm{dc}, \text { aft }}=2 \mathrm{P}_{\mathrm{out}} \cdot\left(\mathrm{PAPR}_{\mathrm{bef}}-\mathrm{PAPR}_{\mathrm{aft}}\right)$

Notice that power savings depend on the operating amplifier output power. This average output power is often limited by some government regulations. For example, as mentioned in [17], the US FCC (Federal Communications Commission) specifies the effective isotropic radiated power (EIRP) not more than 4Watts in unlicensed ISM (Industrial, Scientific and Medical) band. The EIRP also includes antenna gain, which can be between $2 \mathrm{~dB}(1.6)$ and $8 \mathrm{~dB}$ (6.3) for portable devices [25]. The average output power in terms of PAPR can be written as,

$$
\mathrm{P}_{\text {out }}=\frac{4 \text { Watts }}{\mathrm{G}_{\mathrm{a}} \cdot \mathrm{PAPR}}
$$

With the antenna gains of $2 \mathrm{~dB}$ and $8 \mathrm{~dB}$, and representative PAPR $=10 \mathrm{~dB}(10), \mathrm{P}_{\text {out }}$ of $250 \mathrm{~mW}$ and $63 \mathrm{mw}$ are necessary respectively to produce an EIRP of 4 Watts.

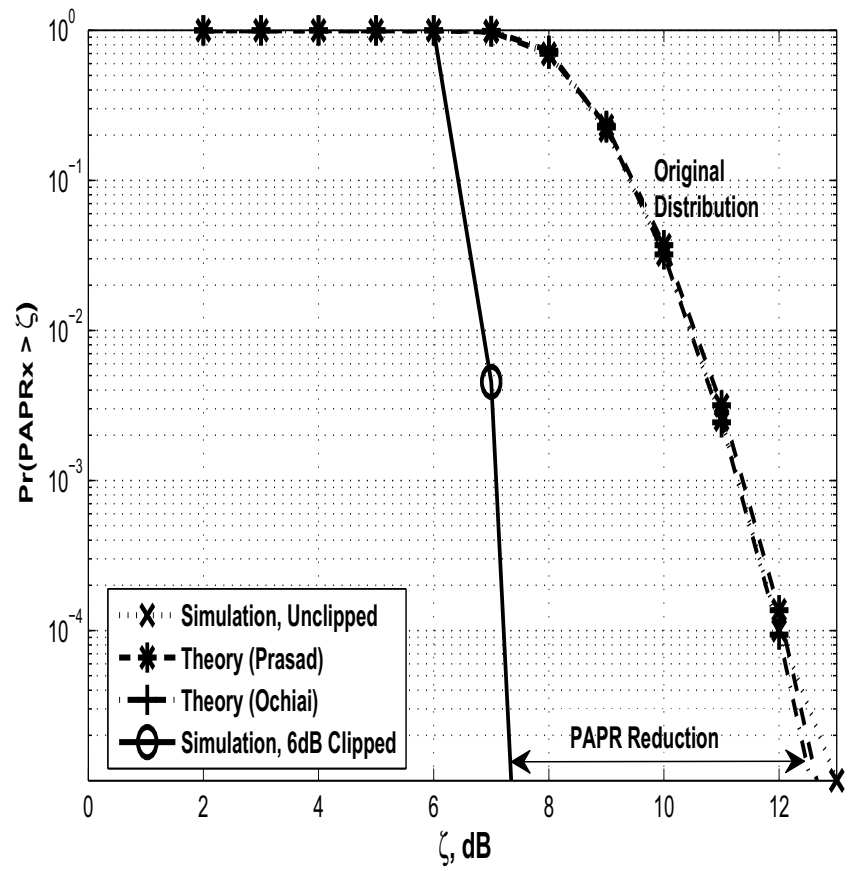

Fig. 3. $\mathrm{CCDF}$ for $6 \mathrm{~dB} \mathrm{ICF}$ (3 iterations)

\section{Power Costs Analysis of Iterative And FILTERING METHOD}

Consider a fixed-point digital-signal-processor (DSP) with parameters as shown in the Table II [17].

The energy consumption per cycle and per N-point FFT/IFFT respectively are

$$
\text { Energy } / \text { cycle }=0.33 \frac{\mathrm{mA} . \mathrm{sec}}{\text { Mcycle }} \cdot 1.26 \mathrm{~V}=415.8 \frac{\mathrm{pWsec}}{\text { cycle }}
$$

TABLE II

RELEVANT DATA FOR FIXED-POINT DSP

\begin{tabular}{cc}
\hline \hline Parameter & Value \\
\hline Current/Processor cycle/Second & $0.33 \mathrm{~mA} / \mathrm{MHz}$ \\
Supply voltage & $1.26 \mathrm{~V}$ \\
Processor frequency & $200 \mathrm{MHz}$ \\
Cycles/256-point FFT & 4786 \\
Cycles/Radix 2 FFT core & 5 \\
Overhead cycles/FFT & 306 \\
Cycles/N-point FFT & $306+5 \frac{N}{2} \log _{2}\left(\frac{N}{2}\right)$ \\
Multiplications/Cycle & 2 \\
Additions/Cycle & 4 \\
Cycles/Complex Multiplications & 3 \\
\hline
\end{tabular}

$$
\begin{aligned}
\text { Energy } / \mathrm{N}-\text { point } & =415.8 \cdot\left[306+5 \frac{\mathrm{N}}{2} \log _{2} \frac{\mathrm{N}}{2}\right] \mathrm{nJ} \\
& =\left[127.2+1.04 \mathrm{Nlog}_{2} \frac{\mathrm{N}}{2}\right] \mathrm{nJ}
\end{aligned}
$$

Referring to Fig. (1), it is obvious that the FFT/IFFT and clipping blocks contribute most of the computational cost. The table III shows the main steps of digital clipping. The clipping threshold is $6 \mathrm{~dB}$. From the Fig. (3), the probability of signal peaks exceeding PAPR of $6 \mathrm{~dB}$ is almost 1 , so the number of complex multiplications (line 3) required is equal to $\mathrm{JN}$. In addition, we assume each 'loop' and 'if' statements incur one cycle, therefore totalling $2 \mathrm{JN}$ cycles per clipping iteration. Finally, we take another JN cycles as overhead cost. The table IV summarizes the ICF computational costs.

TABLE III Steps for Digital Clipping

\begin{tabular}{c}
\hline \hline loop $\mathrm{n}=1: \mathrm{JN}$ \\
if $x_{n}>=$ threshold \\
$x_{n}=$ threshold $\cdot \exp \left(\mathrm{j} x_{n}\right)$ \\
else \\
$x_{n}=x_{n}$ \\
end if \\
end loop
\end{tabular}

TABLE IV

OPERATIONS NECESSARY FOR ICF

\begin{tabular}{cc}
\hline \hline Operations & Value \\
\hline K, iterations & 3 \\
Oversampling Factor, $\mathrm{J}$ & 5 \\
FFT/IFFT & $\mathrm{K}+1$ \\
Multiplications & 0 \\
Additions & 0 \\
Complex Multiplications/iteration & $\mathrm{JN}$ \\
Overhead cycles/iteration & $3 \mathrm{JN}$
\end{tabular}

Using Equations (19) and (20), and Table IV, total computational cost can be given as,

Totalcycles $=7 \cdot\left[306+5 \frac{\mathrm{JN}}{2} \log _{2} \frac{\mathrm{JN}}{2}\right]+3 \cdot(3 \mathrm{JN}+3 \mathrm{JN})$ 
The computational costs in Watts for different ovesampling factor are shown in table V. For comparison purposes, it is assumed that both the power amplifier and the DSP work for the same amount of time so that the computational cost can be written in Watts instead of Joule. Note that the major component of computational cost comes from FFT/IFFT operations (for example, with $\mathrm{J}=5$, FFT/IFFT takes around $90 \%$ of total cost ). The oversampling factor has big impact on processing cost. As mentioned earlier in section II, J $\geq 4$ to capture and clip all the continuous signal peaks. However, the costs are in $\mu W$ which is very small compared to the power savings achieved through PAPR reduction as shown below.

The net power savings becomes (from Equations (17), (19) and (21)),

$$
\mathrm{P}_{\text {netsavings }}=\left\{\begin{array}{l}
2 \mathrm{P}_{\mathrm{out}} \cdot\left(\mathrm{PAPR}_{\mathrm{bef}}-\mathrm{PAPR}_{\mathrm{aft}}\right)- \\
7 \cdot\left[306+5 \frac{\mathrm{JN}}{2} \log _{2} \frac{\mathrm{JN}}{2}\right]+3 \cdot(3 \mathrm{JN}+3 \mathrm{JN}) \\
\times 415.8 \frac{\mathrm{pWsec}}{\text { cycle }}
\end{array}\right.
$$

With $\mathrm{J}=5, \zeta=6 \mathrm{~dB}, \gamma(\mathrm{p})=10^{-5}$, the PAPR reduction is 12.15 (linear scale). The savings for $\mathrm{P}_{\text {out }}$ of nominal $120 \mathrm{~mW}$ would be $2.92 \mathrm{~W}$, which is $68.54 \%$ of power required before PAPR reduction $\left(\mathrm{PAPR}_{\mathrm{bef}}=12.5 \mathrm{~dB}(17.78)\right.$ gives $\left.\mathrm{P}_{\mathrm{dc}, \text { bef }}=4.26 \mathrm{~W}\right)$. Net savings would be $2.92 \mathrm{~W}-96 \mu \mathrm{W} \cong$ $2.92 \mathrm{~W}$. With less conservative clipping probability of $10^{-4}$, the net savings comes to $2.31 \mathrm{~W}, 64.16 \%$ of original power consumption. Note that the actual power savings would vary with processor models.

TABLE V

\section{COMPUTATION COST FOR ICF (3 ITERATIONS)}

\begin{tabular}{ccccc}
\hline \hline$J$ & $F F T$ & Others & TotalCycles & Powercost, $\mu W$ \\
\hline 1 & 31666 & 4608 & 36274 & 15.09 \\
2 & 71986 & 9216 & 81202 & 33.78 \\
3 & 115690 & 13824 & 129510 & 53.87 \\
4 & 161586 & 18432 & 180018 & 74.88 \\
5 & 209120 & 23040 & 232160 & 96.57 \\
\hline
\end{tabular}

\section{CONCLUSION}

The paper analyzed the power savings due to PAPR reduction by clipping and filtering method. The savings takes account of improvement in power amplifier efficiency because of PAPR reduction. The net power savings are shown to be in the order Watts with this simplest PAPR reduction technique. For large clipping ratio $(\geq 6 \mathrm{~dB})$, clipping and filtering method provides an excellent trade-off.

\section{ACKNOWLEDGMENT}

The authors would like to thank anonymous reviewers for their insightful comments and suggestions which improved the presentation of the paper.

\section{REFERENCES}

[1] X. Li and L. J. Cimini, Jr., "Effects of Clipping and Filtering on the Performance of OFDM," IEEE Communications Letters, vol. 2, no. 5, pp. 131-133, May 1998.

[2] J. Armstrong, "Peak-to-average power reduction for OFDM by repeated clipping and frequency domain filtering," IEE Electronics Letters, vol. 38, pp. 246-247, February 2002.

[3] S.H. Leung, S.M. Ju and G.G. Bi, "Algorithm for repeated clipping and filtering in peak-to-average power reduction for OFDM," IEEE Electronics Letters, vol. 38, no. 25, pp. 1726-1727,December 2002.

[4] L. Q. Wang and C. Tellambura, "A Simplified Clipping and Filtering Technique for PAR Reduction in OFDM Systems," IEEE Signal Processing Letters, vol. 12, no. 6, pp.453-456, June 2005.

[5] S. C. Thompson, J. G. Proakis, and J. R. Zeidler, "The Effectiveness of Signal Clipping for PAPR and Total Degradation Reduction in OFDM Systems," IEEE GLOBECOM, pp. 2807-2811, December 2005.

[6] R. V. Nee and R. Prasad, "OFDM for Wireless Multimedia Communications," Artech House Publishers, 2000.

[7] H. Ochiai, "Performance Analysis of Peak Power and Band-Limited OFDM System With Linear Scaling," IEEE Trans. Wireless Communications, vol. 2, no. 5, pp. 1055-1065, September 2003.

[8] S. H. Han and J. H. Lee, "An overview of peak-to-average power ratio reduction techniques for multicarrier transmission," IEEE Wireless Communications, vol. 12, issue 2, pp. 56- 65, April 2005.

[9] H. Ochiai and H. Imai, On the Distribution of the PeaktoAverage Power Ratio in OFDM Signals, IEEE Trans. Commun., vol. 49, no. 2, pp. 28289, Feb. 2001.

[10] Tao Jiang and Yiyan Wu, "An Overview: Peak-to-Average Power Ratio Reduction Techniques for OFDM Signals," IEEE Trans. Broadcasting, Vol. 54, No. 2, pp. 257-268, June 2008.

[11] D. Wulich and L. Goldfeld, "Reduction of Peak Factor in Orthogonal Multicarrier by Amplitude Limiting and Coding," IEEE Trans. Commun., Vol. 47, No.1, pp. 18-21, Jan. 1999.

[12] H. Ochiai and H. Imai, "Performance Analysis of Deliberately Clipped OFDM Signals," IEEE Trans. Commun., Vol. 50, No. 1, pp. 89-101, Jan. 2002.

[13] Arun K. Gurung, Fawaz S. Al-Qahtani, Amin Z. Sadik and Zahir M. Hussain, "One-Iteration-Clipping and Filtering (OICF) Method for PAPR Reduction of OFDM Signals," to appear in ATC Proceedings, Hanoi, Vietnam, Oct. 2008

[14] H. Chen and A. M. Haimovich, "Iteration estimation and cancellation of clipping noise for OFDM signals," IEEE Commun. Letter, Vol. 7, No 7, pp. 305-307, Jul. 2003

[15] N. Y. Ermolova, "Nonlinear amplifier effects on clipped-filtered multicarrier signals," IEE Proc.-Commun., Vol. 153, No. 2, April 2006.

[16] T. Jiang, M. Guizani, H. S. Chen, W.D. Xiang and Y. Y. Wu, "Derivation of PAPR Distribution for OFDM Wireless Systems Based on Extreme Value Theory," IEEE Trans. Wireless Commun., vol. 7, no. 4, April 2008

[17] R. J. Baxley and G. T. Zhou, Power savings analysis of peak-to-average power ratio reduction in OFDM, IEEE Trans. Consumer Electronics, vol. 50, pp. 792798, Aug. 2004

[18] Dov Wulich, "Definition of Efficient PAPR in OFDM," IEEE Commun. Letters, Vol. 9, No. 9, Sept. 2005.

[19] Gill R. Tsouri and Dov Wulich, "Capacity Analysis and Optimization of OFDM with Distortionless PAPR Reduction," to be published soon.

[20] Yaron Handali, Itamar Nizan and Dov Wulich, "On channel capacity of OFDM with SLM method for PAPR reduction," IEEE 24th Convention of Electrical and Electronics Engineers in Israel, pp. 138-140,2006.

[21] E. W. Weisstein, "Lambert W-Function", http://mathworld.wolfram.com/ LambertW-Function.html, accessed June 2008.

[22] J. Tellado, "Multicarrier Modulation with Low PAPR: Applications to DSL and Wireless," Kluwer Academic Publishers, 2000.

[23] S. C. Cripps, RF Power Amplifiers for Wireless Communications, Norwood, MA, Artech, 2006.

[24] N. Chen, "Bandwidth Efficiency and Power Efficiency Issues for Wireless Transmissions," PhD Thesis, Georgia Institute of Technology, USA, 2006.

[25] T. Rappaport, Wireless Communications, Prentice Hall, 2002. 